\title{
INVESTIGATION OF ITD SYMMETRY IN MEASURED HRIRS
}

\author{
Andrea F. Genovese, Jordan Juras, Chris Miller, Agnieszka Roginska
}

\author{
New York University \\ Music and Audio Research Lab \\ 35 West 4th Str. 10012, New York, NY, United States \\ genovese@nyu.edu
}

\begin{abstract}
The Interaural Time Difference is one of the primary localization cues for 3D sound. However, due to differences in head and ear anthropometry across the population, ITDs related to a sound source at a given location around the head will differ from subject to subject. Furthermore, most individuals do not possess symmetrical traits between the left and right pinnae. This fact may cause an angle-dependent ITD asymmetry between locations mirrored across the left and right hemispheres. This paper describes an exploratory analysis performed on publicly available databases of individually measured HRIRs. The analysis was first performed separately for each dataset in order to explore the impact of different formats and measurement techniques, and then on pooled sets of repositories, in order to obtain statistical information closer to the population values. Asymmetry in ITDs was found to be consistently more prominent in the rear-lateral angles (approximately between $90^{\circ}$ and $130^{\circ}$ azimuth) across all databases investigated, suggesting the presence of a sensitive region. A significant difference between the peak asymmetry values and the average asymmetry across all angles was found on three out of four examined datasets. These results were further explored by pooling the datasets together, which revealed an asymmetry peak at $110^{\circ}$ that also showed significance. Moreover, it was found that within the region of sensitivity the difference between specular ITDs exceeds the just noticeable difference values for perceptual discrimination at all frequency bands. These findings validate the statistical presence of ITD asymmetry in public datasets of individual HRIRs and identify a significant, perceptually-relevant, region of increased asymmetry. Details of these results are of interest for HRIR modeling and personalization techniques, which should consider implementing compensation for asymmetric ITDs when aiming for perceptually accurate binaural displays. This work is part of a larger study aimed at binaural-audio personalization and user-characterization through non-invasive techniques.
\end{abstract}

\section{INTRODUCTION}

One of the most crucial binaural cues in spatial sound is the Interaural Time Difference (ITD). For a particular location in space, an ITD describes the difference of time-of-arrival between the two ears for a sound source's direct path. ITDs are an important factor in how the human brain localizes a sound source and$$
\text { (c) (i) (2) }
$$

This work is licensed under Creative Commons Attribution $Ð$ Non Commercial 4.0 International License. The full terms of the License are available at http://creativecommons.org/licenses/by-nc/4.0
}

it is the primary binaural cue for low frequencies [1]. ITDs are contained within measured Head-Related Impulse Responses (HRIRs), which can be used to transfer the perceptual cues of a sound source at some point in space about a listener's head to any mono sound file through time-domain convolution [2].

It is generally accepted that a users' experience is significantly improved by the use of individually measured HRIRs rather than the HRIRs recorded on mannequin dummy heads [2]. Different pinnae and head morphologies affect both ITD and spectral cues individually for each user. Only listeners with a morphology close to that of the mannequin will experience a satisfactory binaural reproduction, while most listeners will experience a degraded spatial auditory image.

Recently, many modeling techniques for parameterizing individual HRTFs (the Fourier transformation of HRIRs) from pictures and scans came to the attention of the sound engineering community [3] [4]. One such technique [5], generates a measure of the user's head size through a photographic technique and a subsequent adaptation of a general HRIR subset by the insertion of the user's head-size related ITDs, calculated via a spherical head model (first developed by [6]).

However, these models assume a symmetrical morphology of the listener's head and pinnae, implying symmetrical cues for sound localization between the left and right sides of the horizontal plane. This assumption of symmetry presumes an equal ITD value for a source placed, for example, at an azimuthal location of $\theta=-30^{\circ}$ and its respective mirrored-equivalent at $\theta=+30^{\circ}$ in the opposite hemisphere. In literature, there is an indication that this assumption of symmetry does not always hold true [7], motivating the need for more exploratory studies aimed at analyzing and assessing available spatial audio data in order to evaluate the severity of asymmetric ITDs. While Zhong et al. [7] have explored the asymmetry of HRTFs, the scope of this paper will focus on ITDs only.

Evidence of significantly asymmetrical ITDs would motivate further exploration on the correlations between morphological asymmetries and mirrored ITDs. An analysis of this relationship could be of significant value to the future development and improvement of HRIR/HRTFs modeling techniques that could adjust or compensate modeled personalized HRIRs for a user with asymmetric cues. At this stage, no perceptual test which could validate the relevance of physical ITD asymmetries has yet been performed, nonetheless the ITD differences between mirrored locations can be compared to established Just Noticeable Difference (JND) values [8] for location discrimination. Specifically, the goal of this paper is to explore whether ITDs in individual-HRIR public 
databases are statistically asymmetric and whether there is a pattern in the asymmetry across angles in the horizontal plane. This exploratory analysis is part of a bigger study by New York University's Music and Research Laboratory (NYU-MARL), which is focused on extracting personalized spatial audio cues through non-invasive techniques.

\section{DATABASES}

For this analysis, publicly available databases of individual HRIRs were collected. The datasets were chosen on the basis of their frequent use within the field and their diversity in terms of sample rate and measurement methodology. Four databases of individual HRIRs measurements were found to be suitable for this analysis. Due to the different characteristics and measurement methods of the databases, it was decided that the analysis of ITD asymmetry would be preformed separately and independently for each dataset. The four selected datasets are: LISTEN [9], CIPIC [10], FIU [11] and MARL [12].

\subsection{LISTEN database}

This database was recorded for the LISTEN project [9] initiated as a collaboration between IRCAM and AKG. This set was recorded on 51 subjects $^{1}$ at $44.1 \mathrm{kHz}$ sampling rate. The set consists of 187 locations per subject, measured at different azimuth resolutions for 10 respective elevation angles spaced at $15^{\circ}$ from $\phi=-45^{\circ}$ to $+90^{\circ}$.

The advantage of this set lies in its measurement technique which made use of a crane structure, to precisely move a loudspeaker along a rig, and of a software-controlled rotating chair with a headrest used to rotate the subject to the desired azimuth degree. Using a single loudspeaker avoided the issue of having to compensate for each loudspeaker position. In comparison with other datasets in this list, the method used for LISTEN limited the possibility of measurement errors caused by human misalignments with the target angle, which would likely affect ITDs. Furthermore, the microphone capsules used for recording allowed for blocked-meatus conditions to prevent resonance of the ear-canal.

The drawback of the LISTEN database is the $44.1 \mathrm{kHz}$ sampling rate which gives a low temporal resolution for estimating the magnitude of ITD asymmetry (distance between samples is $22 \mu \mathrm{s}$ as opposed to $10 \mu \mathrm{s}$ in the $96 \mathrm{kHz}$ case).

\subsection{CIPIC database}

The CIPIC database [10] was compiled and made publicly available by UC Davis. This set consists of 25 azimuth locations recorded between $-80^{\circ}$ to $+80^{\circ}$ in azimuth and 50 different elevations from $-45^{\circ}$ to $+230.625^{\circ}$ (steps of $5.625^{\circ}$ ), for a total of 1250 locations recorded per subject (45 subjects). HRTFs were measured using Golay-code signals at $44.1 \mathrm{kHz}$, where the subject seated in a $1 \mathrm{~m}$ radius hoop aligned to the subject's interaural axis. Subjects were not constrained but were able to monitor their head position.

One particularly interesting aspect of this measurement set is the inclusion of a detailed collection of anthropometric data of head and pinna morphology parameters which allows for the possibility of a future correlational study between ITD asymmetries

\footnotetext{
1 "Subject 1034" was later removed from the set due to inconsistencies in measurement data. So, 50 subjects were ultimately considered.
}

and anthropomorphic asymmetries. Pinna measurements were collected on both sides of the head, providing useful information about morphological asymmetries within subjects' ear characteristics. However, the CIPIC dataset was constructed using a nonoptimal sample rate $(44.1 \mathrm{kHz})$ for a high-precision detection of onsets.

\subsection{FIU database}

The Florida International University DSP Lab measured the individual HRTFs of 15 different subjects ${ }^{2}$ at 12 azimuth locations $\left(30^{\circ}\right.$ spacing) and 6 elevations [11]. Recordings were conducted at $96 \mathrm{kHz}$ and were accompanied by anthropometric data measured via a 3D scan of the pinnae. The set includes measurements for 6 different elevations spaced at $18^{\circ}$ apart from $-36^{\circ}$ to $54^{\circ}$. The measurements were conducted using the HeadZap system from AuSIM 3D using Golay-Code. The recording methodology relied on a rotating chair and a laser pointer to align the subject, and is thus not fully reliable as tiny head movements between measurements might give rise to ITD asymmetries. It was found that one of the angle mirrored pairs $\left( \pm 150^{\circ}\right)$ had to be dropped due to problems in the set composition where the HRIR data from $-150^{\circ}$ was identical to that at $+150^{\circ}$.

\subsection{MARL database}

The last database to be analyzed was the NYU MARL (Music and Audio Research Lab) dataset collected by Andreopolou et al. [12] in 2013 and formatted to the MARL repository standard described in [13]. Four participating subjects had their personal HRTFs measured ten times each using different alignment methods (rotating stool with laser pointer or magnetic tracker) at a $48 \mathrm{kHz}$ sampling rate. The MARL dataset thus allows for the study of HRTF feature variability across measurements. HRTFs were measured with a resolution of $10^{\circ}$ azimuth and $15^{\circ}$ elevation angles (going from $-30^{\circ}$ to $+30^{\circ}$ of elevation) for a total of 180 filter pairs per set. 40 sets (10 sets per 4 subjects) were collected - reduced to 32 after removal of corrupted sets. HRTFs were recorded with different techniques across repeated measurements using Golay Code, Maximum Length Sequences, and sine sweep signals.

In this paper, each repeated measurement on the same subject was treated as if it were a different independent subject, thus pooling together different measurement techniques within the dataset. The shortcomings of this dataset for our purposes are due to the low-precision of the alignment techniques and non-uniformity of the measurement method. These factors make it hard to discern between the presence of asymmetry in ITD and simple misalignments due to head movements during recording. These key aspects make MARL a very different dataset that has to be interpreted more carefully than the rest. The actual number of subjects is low, so a low variance of asymmetry is expected. However, it is interesting to explore how the non-uniformity of techniques will impact measurement error and therefore the results.

\section{ANTHROPOMETRIC ASYMMETRY}

Table 1 illustrates a pre-analysis exploration on the symmetry of individuals' anthropometric data included in the CIPIC database for a sample of their measured subjects. The provided data [10] includes a variety of pinnae measurements for both the left and

\footnotetext{
${ }^{2}$ Later reduced to 14 due to a formatting problem
} 


\begin{tabular}{|l|ll|}
\hline Anthropometric Feature & Mean & $\sigma$ \\
\hline Cavum Concha Height $(\mathrm{cm})$ & 0.1213 & 0.1077 \\
Cymba Concha Height $(\mathrm{cm})$ & 0.0910 & 0.0642 \\
Cavum Concha Width $(\mathrm{cm})$ & 0.1357 & 0.0883 \\
Fossa Height $(\mathrm{cm})$ & 0.1551 & 0.1421 \\
Pinna Height $(\mathrm{cm})$ & 0.3207 & 0.2737 \\
Pinna Width $(\mathrm{cm})$ & 0.1613 & 0.1251 \\
Intertragal Incisure Width $(\mathrm{cm})$ & 0.0715 & 0.0551 \\
Cavum Concha Depth $(\mathrm{cm})$ & 0.1295 & 0.1152 \\
Pinna Rotation Angle $\left(\mathrm{deg}^{\circ}\right)$ & 0.0957 & 0.0415 \\
Pinna Flare Angle $\left(\mathrm{deg}^{\circ}\right)$ & 0.0813 & 0.0638 \\
\hline
\end{tabular}

Table 1: Mean and Standard deviation of the left-right difference of pinnae features measurements for 37 subjects in CIPIC

the right hemisphere. The table reports the values of the average feature difference between the left and right ear measure across subjects, and the standard deviation of these differences. The most varied feature across subjects is the "Pinna Height", with an average difference of $0.3207 \mathrm{~cm}$. The presence of these morphological asymmetries supports the hypothesis that ITDs will also be asymmetrical.

\section{ANALYSIS AND RESULTS}

All of the available data was analyzed to produce a series of plots intended to illustrate the presence and severity of asymmetry specific to ITDs. Due to the mismatch in angle resolutions, sample rate, and reliability of measurement techniques, the first-stage of the analysis did not pool the data but was rather conducted separately for each database. Each set is therefore analyzed in its own context, allowing us to observe whether different measurement methods yield different results.

In the context of this document, the ITD was calculated as a measure in samples (then translated into seconds) between the two points of maximum time-domain cross-correlation, between the left and right ear HRIR signals. For consistency, the ITD cross-correlation calculation function was applied to each database, including those who already provided ITD data. This was not possible, however, for the FIU database, as the HRIR measurements were only available in a minimum phase format therefore the provided ITD values were used. To account for clear measurement errors, a pre-analysis inspection of the data led to the discarding of strong outlier measurements that could affect the results.

The ITD symmetry for every subject was calculated as the absolute value of the difference of ITD magnitude with that of their respective mirrored counterpart. A difference of 0 would indicate complete symmetry between hemispheres, while a higher absolute difference would point to a higher level of asymmetry. The values were averaged across each set of $N$ subjects for every available angle $\theta \in[0 ; 180]$ within each dataset:

$$
\bar{S}(\theta)=\frac{1}{N} \sum_{n \in N}|| I T D_{n}(\theta)|-| I T D_{n}\left(360^{\circ}-\theta\right) \|
$$

The mean and standard deviation of the absolute asymmetry across subjects was measured for those angles on the horizontal plane that possessed an ITD value at both hemispheres. Thus, the $0^{\circ}$ and $180^{\circ}$ angles were excluded from the analysis as they lack a mirrored correspondent across hemispheres.

\begin{tabular}{|l|llll|}
\hline DATASET & $\mathrm{N}$ & $\theta_{P}$ & $\bar{S}\left(\theta_{P}\right)$ & $\bar{S}^{\prime}$ \\
\hline LISTEN (a) & 50 & $105^{\circ}$ & $84 \mu s$ & $51 \mu \mathrm{s}$ \\
CIPIC (b) & 45 & $115^{\circ}$ & $119 \mu \mathrm{s}$ & $49 \mu \mathrm{s}$ \\
FIU (c) & 14 & $120^{\circ}$ & $72 \mu \mathrm{s}$ & $32 \mu \mathrm{s}$ \\
MARL (d) & $31(4)$ & $150^{\circ}$ & $47 \mu \mathrm{s}$ & $35 \mu \mathrm{s}$ \\
D1 (a+b+d) & 126 & $110^{\circ}$ & $80 \mu \mathrm{s}$ & $45 \mu \mathrm{s}$ \\
D2 (a+b) & 95 & $110^{\circ}$ & $94 \mu \mathrm{s}$ & $49 \mu \mathrm{s}$ \\
\hline
\end{tabular}

Table 2: Peak ITD asymmetry values, peak positions across examined databases and non peak curve averages

\begin{tabular}{|l|lll|}
\hline DATASET & df & $t$ & $p$ \\
\hline LISTEN (a) & 49 & 3.91 & $\mathbf{1 . 6} \boldsymbol{e}-\mathbf{0 4}$ \\
CIPIC (b) & 44 & 3.90 & $\mathbf{3 e - 0 3}$ \\
FIU (c) & 13 & 3.27 & $\mathbf{1 . 4 e - 0 4}$ \\
MARL (d) & 30 & 1.51 & 0.07 \\
D1 (a+b+d) & 125 & 4.46 & $\mathbf{8 . 9 e - 0 6}$ \\
D2 (a+b) & 94 & 4.70 & $\mathbf{4 . 4 e - 0 6}$ \\
\hline
\end{tabular}

Table 3: One-tail t-test results. Three out of four datasets (in boldface) showed significance at $95 \%$ confidence level.

The plots in Figure 1 illustrate the average ITD difference across subjects between the left and right hemispheres on the horizontal plane $\left(0^{\circ}\right.$ elevation $)$ for each dataset. Error bars show the 95\% confidence interval boundaries for each angle. In Table 2, the peaks of the ITD average curves are reported to the nearest microsecond, along with the associated azimuth angles. Ignoring the MARL set, once notices the proximity of the peak azimuths placed around $110^{\circ} \pm 10^{\circ}$. In the case of MARL, no clear peaks can be identified and the curve looks somewhat flatter. 


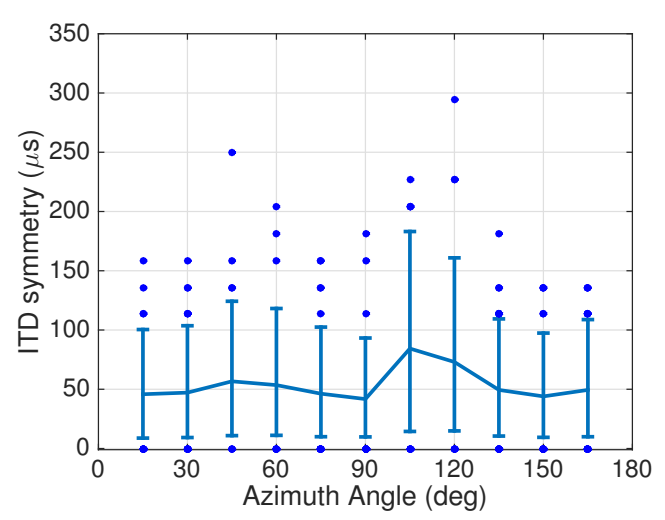

(a) LISTEN

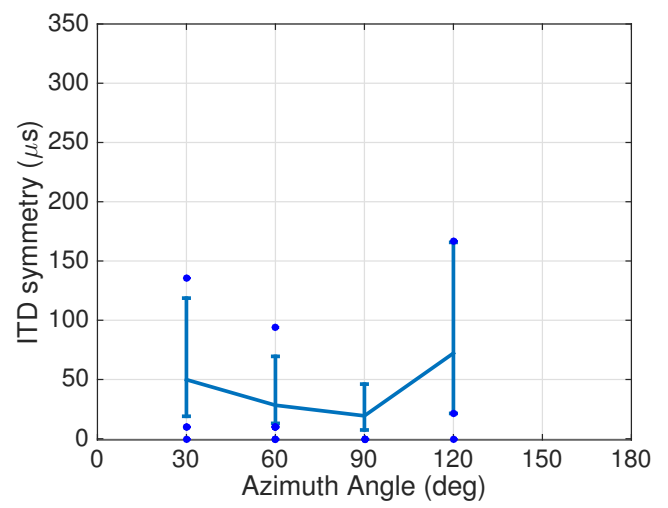

(c) FIU

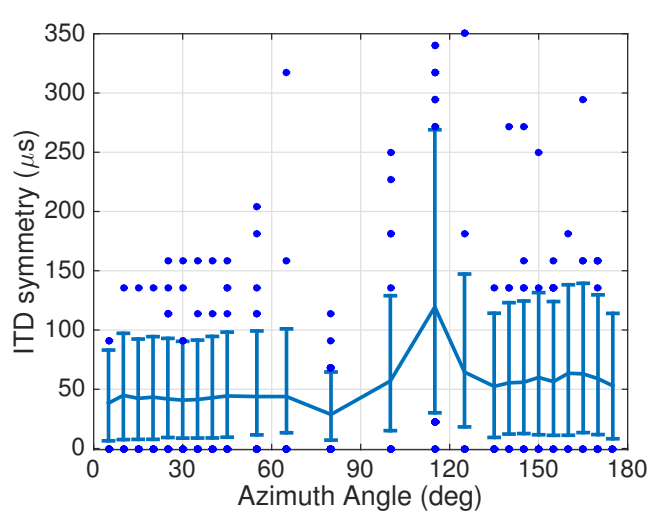

(b) CIPIC

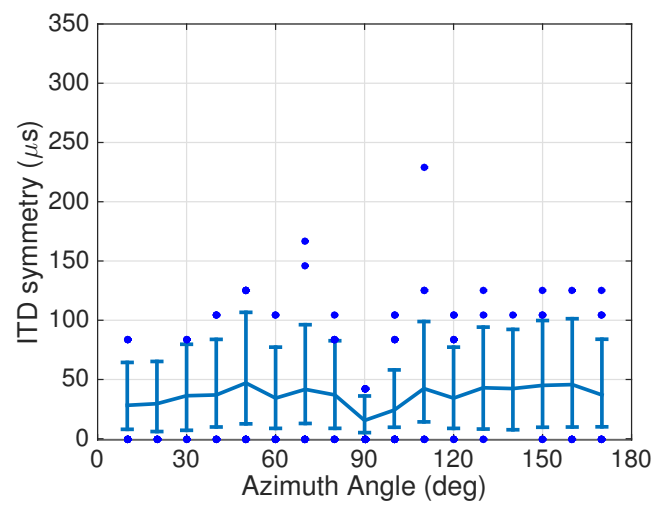

(d) MARL

Figure 1: Average ITD symmetry and standard deviation across subjects for the available datasets calculated using (1). The azimuthal resolution depended on the set. All the ITDs were taken at zero elevation.

In Figure 2, we notice a region of higher ITD asymmetry magnitude. For three out of the four examined databases, this region ranges roughly in the lower bound around $90^{\circ}$ and $130^{\circ}$ in the upper bound. Unfortunately, the precision of this statement is undermined by the absence of coherent resolution and sample rates between databases, though the general shape of the asymmetry curves across databases is similar (Figure 2).

The presence of this region motivates further exploration into whether HRIRs at certain angles are more sensitive to morphological asymmetries than others. To inspect this hypothesis, one-tailed t-tests between the ITD symmetry values at the peak azimuth $\theta_{P}$ for each subject, and the curve's average asymmetry value excluding the peak $\bar{S}^{\prime}$, were conducted for each set (details in Table 2). The results of this test highlight whether the asymmetry at the peak is significantly higher than the asymmetry at the rest of the angles, with significant difference show for three of the sets at $95 \%$ confidence $(p<.05)$ (Table 3$)$.

From Figure 2 we also note a consistent minimum ITD asymmetry at $90^{\circ}$ azimuth indicating a possible interaction with the angle of incidence.

\subsection{Data Pooling}

After observing the results for each database, the data was pooled in order to extract ITD asymmetries representative of the entire sample population and increase confidence in the results obtained thus far. The calculated ITDs for the databases were linearly interpolated to the whole $360^{\circ}$ horizontal plane. The FIU set was excluded due to excessively missing data. Since the MARL dataset consists of repeated measurements for four subjects and its measurement techniques are less reliable from CIPIC and LISTEN, two versions of data-pooling sets were created, one with MARL (D1, $\mathrm{N}=126)$ and one without MARL (D2, $\mathrm{N}=95){ }^{3}$

Results in Figure 3 show that, regardless of the inclusion of the MARL set, the asymmetry peak is centered at $110^{\circ}$. In both cases, the t-tests values shown in Table 2 show a significant difference (95\% confidence level) of the peak region's asymmetry against all other values.

\footnotetext{
${ }^{3}$ ITD angle-alignment based on Left-Right contours was considered to correct for misalignment, however that would have meant to assume a spherical head model. Preliminary tests showed that the asymmetry magnitudes were not reduced by the alignment, on the contrary they increased.
} 


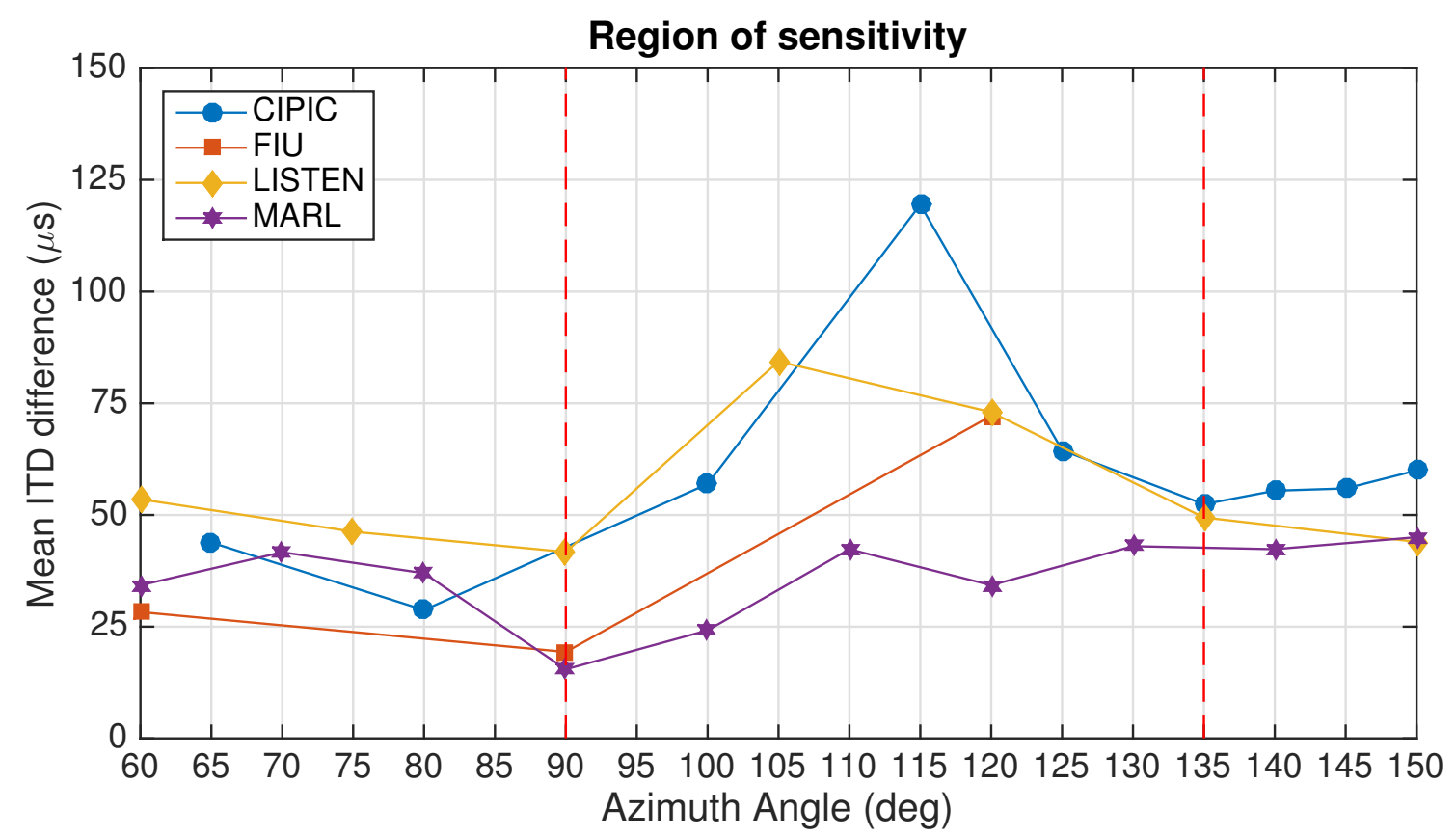

Figure 2: Close-up superposition of all mean ITD asymmetry curves across $60^{\circ}$ and $150^{\circ}$. An identified region of sensitivity spans between $90^{\circ}$ and $130^{\circ}$
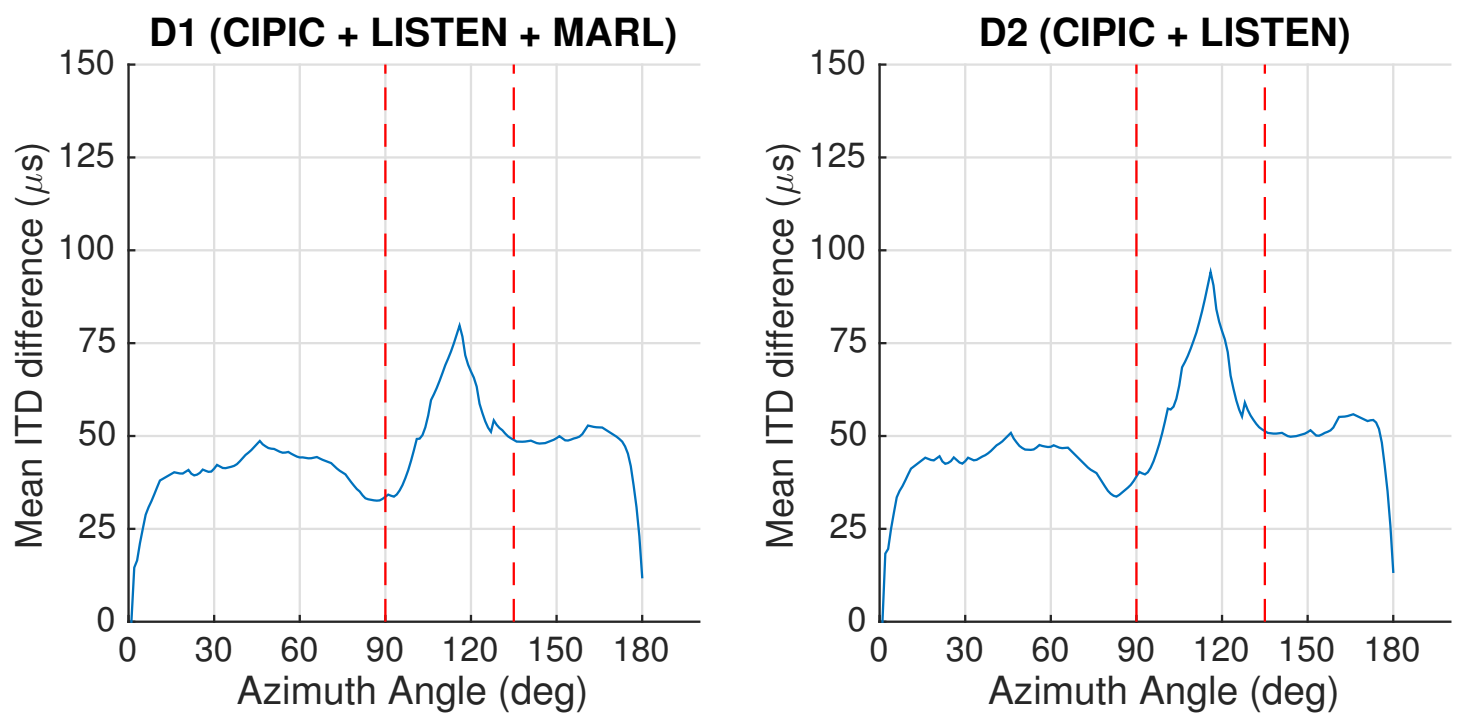

Figure 3: Average asymmetry across the horizontal plane for pooled datasets D1 (CIPIC + LISTEN + MARL) and D2 (CIPIC + LISTEN). The dotted lines represent the sensitivity region

\subsection{Summarization Table}

Table 4 illustrates an attempt to summarize and characterize the nature of the ITD asymmetry curve of each database and parameterize its distribution across the horizontal plane. The table shows values approximated to the nearest microsecond for the grand mean, standard error, skewness and kurtosis. The standard error for the FIU database is much greater due to the smaller number of subjects. Table 4 provides a quick reference of the severity of the asymmetry in all databases. The skewness value describes the off- set of the sensitivity region, while the kurtosis indicates its width.

\section{DISCUSSION}

The previous section identifies a "region of sensitivity" located between $90^{\circ}$ and $130^{\circ}$ azimuth, whose presence is validated by the fact that all datasets showing a significant difference between the peak asymmetry values and the average asymmetry values find their peaks located within $15^{\circ}$. The MARL dataset is the only 


\begin{tabular}{|l|llllll|}
\hline Dataset & $N$ & Mean & Median & SE & Skewness & Kurtosis \\
\hline LISTEN (a) & 50 & $54 \mu s$ & $50 \mu s$ & $1.8 \mu s$ & 1.43 & 3.756 \\
CIPIC (b) & 45 & $52 \mu s$ & $49 \mu s$ & $2.6 \mu s$ & 2.524 & 11.087 \\
FIU (c) & 14 & $42 \mu s$ & $39 \mu s$ & $6.3 \mu s$ & 0.341 & N/A \\
MARL (d) & 31 & $36 \mu s$ & $37 \mu s$ & $1.5 \mu s$ & -0.946 & 3.142 \\
D1 (a+b) & 126 & $46 \mu s$ & $45 \mu s$ & $1 \mu s$ & 0.407 & 4.754 \\
D2 (a+b+d) & 95 & $49 \mu s$ & $47 \mu s$ & $1.3 \mu s$ & 1.032 & 5.395 \\
\hline
\end{tabular}

Table 4: Summarization of symmetry curves in each database

dataset showing non-significant results, but is less suited to explore population asymmetry parameters due to its focus on a small number of subjects. It is, however, rather suited for exploring asymmetry caused by measurement error. The four MARL subjects display more symmetric features on average, though no morphological data is provided to verify this. The lack of strong asymmetry peaks in MARL may result from the small pool of subjects, and seems to be independent of measurement method.

indicate that measurement errors are not the cause for the strong asymmetries in the other datasets. We therefore hypothesize that the asymmetry observed in the three other sets is independent of the measurement technique.

One reason to keep the datasets separate was to observe whether different measurement techniques have an impact on the magnitude of ITD asymmetry. The pooled data looked for more general statistics regarding average asymmetries and the sensitivity region that would approach a representative population curve. As depicted in Figure 3, a significant peak occurs at $\theta= \pm 110^{\circ}$, which can be regarded as the point of maximum asymmetry in the azimuth plane - observed within the proposed region of sensitivity. For D1 (MARL set included), the average asymmetry was about $45 \mu \mathrm{s}$ and the peak asymmetry $80 \mu \mathrm{s}$. Excluding MARL (D2) the average asymmetry was $47 \mu \mathrm{s}$ and the peak asymmetry $94 \mu \mathrm{s}$. T-tests for both cases showed significant difference between peak and average asymmetry $(p<.05)$. The inclusion of the MARL database did not have a significant impact on the results.

Is the magnitude of the ITD asymmetry large enough to cause perceptual asymmetry? Per Klumpp and Eady [8], the ITD's JND is highly frequency-dependent. The study found that for a $1 \mathrm{kHz}$ pure tone the JND was on average about $11 \mu \mathrm{s}$, but for a $90 \mathrm{~Hz}$ tone the JND increased to $75 \mu \mathrm{s}$, while for band-limited random noise the average JND drops to $9 \mu \mathrm{s}$. As depicted in Figure 3, the peak values of the average ITD asymmetry range between $80-94 \mu \mathrm{s}$, exceeding the JND values for all frequency bands. Therefore, the localization error between two mirrored locations is significantly more noticeable in the region of sensitivity. Figures 1 (a) and (b) both present peaks higher than most JNDs. The asymmetry results of Figure 1 (c), and the t-test results are less reliable due to the low number of subjects (14) and error-prone methodology, but nevertheless the curve is indicative of a similar range of sensitivity between $90^{\circ}$ and $130^{\circ}$.

These results are useful for guiding the study of the influence of head and ear morphologies on sound perception. Since the peak asymmetries were found on the rear-lateral angles, it is possible to hypothesize that asymmetrical pinnae might interfere with the direct path of the sound source to the timpanus. A likely factor is illustrated in Table 1 which depicts the Pinna Height as the most asymmetric anthropometric feature in the pool of subjects of CIPIC.

From an engineering perspective, the impact of these findings relates to the trade-off between the complexity and accuracy of a binaural audio reproduction system. For the angles within the sensitivity range, the localization accuracy of a listener is likely to degrade if the system uses standardized non-individual HRIRs or symmetrical-head models for personalization. Depending on the application, localization accuracy might be more or less important for the correct delivery of the intended user experience. Applications aimed for entertainment, such as music or movie reproduction, are unlikely to necessitate precise localization and may prefer a simpler processing environment which assumes a spherical head model. If, instead, localization accuracy is key (i.e. binaural navigation systems or pyschoacoustics studies), then ITD asymmetry compensation should be considered.

\section{CONCLUSIONS}

This study used a number of publicly available personalized HRIR databases to investigate the presence and severity of asymmetric ITD measurement data between the left and right hemisphere on the horizontal plane. A consistent azimuth region of higher sensitivity, approximately between $\pm 90^{\circ}$ to $\pm 130^{\circ}$, was identified in all datasets. T-tests confirmed that the asymmetry in the region's peak is significantly different than the average asymmetry at all other angles for three out of four datasets. These results were further explored by merging the datasets in interpolated pooled repositories, which identified a general ITD peak asymmetry at $110^{\circ}$, also significantly different than the average asymmetry.

From a perceptual standpoint, the asymmetry peaks in set D1 and D2, which range from $80 \mu s$ to94 $\mu s$, are higher than the ITD JND thresholds found in [8]. At least for the examined datasets, the peak asymmetries in the sensitivity region are likely to be severe enough to be noticeable at all frequencies.

These findings validate the statistical presence of perceptuallynoticeable asymmetric ITDs in individually measured subjects. The likely causes of ITD asymmetries are the anthropometric 
asymmetries of the head and ears which lead to morphological differences across hemispheres. These results are of interest for HRIR personalization and modeling techniques that aim for accurate localization, especially if making use of the same public repositories utilized for this study.

\subsection{Further Work}

An immediately apparent next step would expand this analysis to more datasets. It would also be interesting to further explore the role of other variables, such as elevation and measurement technique, in ITD asymmetry. However, the lack of a common format in the used databases created a non-ideal situation where low angle-resolutions made it harder to precisely refine the location of the asymmetry peaks, having to resort to interpolation for missing angles. Moreover, low sampling rates are an obstacle to precise ITD values. It is hoped that in the future more public datasets will adhere to a defined standard such as the SOFA conventions [14], developed as HRTF data exchange format and able to make this type of analysis easily implementable.

Further analysis on the morphological characteristics that give rise to the ITD asymmetry could highlight a possible role of the outer ear pinnae in modifying the direct path from the source to the ear canal. In the context of binaural personalization, there is motivation to establish an exact relationship between anthropometry and sound perception. It would be interesting to run a regression test and look for correlations between physical ITD asymmetry and morphological asymmetry for a pool of subjects representative of the general population, perhaps starting with Pinnae Height (Table 1). Binaural audio models could then improve the listening experience by implementing an additional layer of signal compensation based on the observed anthropometric parameters. For example, if the asymmetry found in the HRIR measurements were to be correlated to morphological asymmetries, the mismatch between hemispheres could be easily parameterized and accounted for by using a more comprehensive individualization technique (i.e. photographic information), using only the identified relevant features. This type of study is currently a challenge due to the limited availability of morphological data across public datasets.

As a final note, the importance of these results has to be assessed in light of the fact that physical asymmetry may not coincide with perceptual asymmetry of spatial sound. In fact, perceptual compensation via neural internal-delay has been previously hypothesized [15] [16]. Formal listening tests are required in order to confirm the indications given by the JND values and to inspect if there is a direct correlation between physical and perceptual asymmetries.

\section{REFERENCES}

[1] F. L. Wightman and D. J. Kistler, "The dominant role of lowfrequency interaural time differences in sound localization," The Journal of the Acoustical Society of America, vol. 91, no. 3, pp. 1648-1661, 1992.

[2] M. Morimoto and Y. Ando, "On the simulation of sound localization." Journal of the Acoustical Society of Japan (E), vol. 1, no. 3, pp. 167-174, 1980.
[3] N. Gupta, A. Barreto, and M. Choudhury, "Modeling head-related transfer functions based on pinna anthropometry," in Proc. of the Second International Latin American and Caribbean Conference for Engineering and Technology (LACCEI), 2004.

[4] M. Dellepiane, N. Pietroni, N. Tsingos, M. Asselot, and R. Scopigno, "Reconstructing head models from photographs for individualized 3d-audio processing," in Computer Graphics Forum, vol. 27, no. 7. Wiley Online Library, 2008, pp. 1719-1727.

[5] J. Juras, C. Miller, and A. Roginska, "Modeling itds based on photographic head information," in Audio Engineering Society Convention 139, Oct 2015.

[6] V. R. Algazi, C. Avendano, and R. O. Duda, "Estimation of a spherical-head model from anthropometry," Journal of the Audio Engineering Society, vol. 49, no. 6, pp. 472-479, 2001.

[7] X.-L. Zhong, F.-c. Zhang, and B.-S. Xie, "On the spatial symmetry of head-related transfer functions," Applied Acoustics, vol. 74, no. 6, pp. 856-864, 2013.

[8] R. Klumpp and H. Eady, "Some measurements of interaural time difference thresholds," The Journal of the Acoustical Society of America, vol. 28, no. 5, pp. 859-860, 1956.

[9] O. Warufsel, "Listen hrtf database, ircam," 2002. [Online]. Available: http://recherche.ircam.fr/equipes/salles/listen/

[10] V. R. Algazi, R. O. Duda, D. M. Thompson, and C. Avendano, "The cipic hrtf database," in Applications of Signal Processing to Audio and Acoustics, 2001 IEEE Workshop on the. IEEE, 2001, pp. 99-102.

[11] N. Gupta, A. Barreto, M. Joshi, and J. C. Agudelo, "Hrtf database at fiu dsp lab," in Acoustics Speech and Signal Processing (ICASSP), 2010 IEEE International Conference on. IEEE, 2010, pp. 169-172.

[12] A. Andreopoulou, A. Rogińska, and H. Mohanraj, "A database of repeated head-related transfer function measurements," 2013

[13] A. Andreopoulou and A. Roginska, "Towards the creation of a standardized hrtf repository," in Audio Engineering Society Convention 131, Oct 2011. [Online]. Available: http://www.aes.org/e-lib/browse.cfm?elib=16096

[14] P. Majdak, Y. Iwaya, T. Carpentier, R. Nicol, M. Parmentier, A. Roginska, Y. Suzuki, K. Watanabe, H. Wierstorf, H. Ziegelwanger, et al., "Spatially oriented format for acoustics: A data exchange format representing head-related transfer functions," in Audio Engineering Society Convention 134. Audio Engineering Society, 2013

[15] S. E. Boehnke and D. P. Phillips, "Azimuthal tuning of human perceptual channels for sound location," The Journal of the Acoustical Society of America, vol. 106, no. 4, pp. 19481955, 1999.

[16] P. Joris and T. C. Yin, "A matter of time: internal delays in binaural processing," Trends in neurosciences, vol. 30, no. 2, pp. 70-78, 2007. 